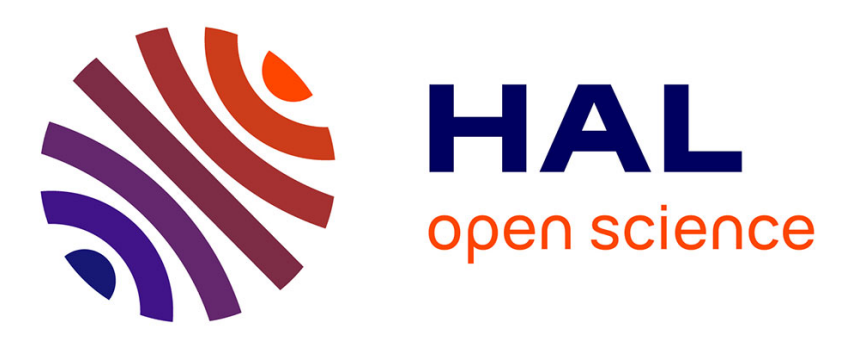

\title{
Massifs boisés de Parcs et de Promenades: Leur consistance - Leur Traitement, Leur régénération
}

Eugène Toussaint

\section{To cite this version:}

Eugène Toussaint. Massifs boisés de Parcs et de Promenades: Leur consistance - Leur Traitement, Leur régénération. Revue forestière française, 1955, 5, pp.351-369. 10.4267/2042/27090 . hal03383789

\section{HAL Id: hal-03383789 \\ https://hal.science/hal-03383789}

Submitted on 18 Oct 2021

HAL is a multi-disciplinary open access archive for the deposit and dissemination of scientific research documents, whether they are published or not. The documents may come from teaching and research institutions in France or abroad, or from public or private research centers.
L'archive ouverte pluridisciplinaire HAL, est destinée au dépôt et à la diffusion de documents scientifiques de niveau recherche, publiés ou non, émanant des établissements d'enseignement et de recherche français ou étrangers, des laboratoires publics ou privés. 


\title{
MASSIFS BOISEES DE PARCS ET DE PROMENADES Leur consistance - Leur traitement Leur régénération
}

\author{
(Leçons à tirer du Bois de Boulogne et du Bois de Vincennes)
}

\begin{abstract}
Associés en massifs plus ou moins étendus et denses, formant ainsi des boqueteaux, des bois, des forêts, les arbres jouent un rôle essentiel dans l'économie d'un pays en lui procurant sous des formes diverses des matériaux indispensables à la société humaine. Et c'est ce point de vue qui a surtout dicté en France l'ordonnance du $\mathrm{I}^{\text {er }}$ août $\mathrm{I} 827$, portant règlement des aménagements des forêts de
\end{abstract} l'Etat.

De nos jours, on ne met plus en doute que la forêt n'est pas moins d'utilité générale par l'heureuse influence qu'elle exerce pour la protection du sol, ainsi que sur le climat, la régularisation du régime des eaux, etc...

Mais ces considérations deviennent négligeables, passent à l'arrière-plan pour le forestier ou le jardinier qui se trouve en présence de massifs boisés, d'origine naturelle ou artificielle, utilisés ou créés pour devenir un des éléments importants d'un parc ou d'une vaste promenade.

Au $\mathrm{XVII}^{\ominus}$ siècle, dans l'architecture du grand siècle, LE NotrE avait, en association avec l'ordonnance des bâtiments de l'époque, conçu le plan du jardin régulier pour la vie mondaine et la parade. Il réservait aux foules suffisamment d'espaces libres, d'allées, de dégagements, pour que les usagers plus désireux alors de se faire voir ou d'assister à un spectacle mondain que de jouir du charme de la nature n'aient pas à chercher en s'aventurant en dehors des chemins, des plateformes et des terrasses la place qui leur aurait fait défaut. Le massif boisé n'était pas fait pour être fréquenté. C'était un cadre qui de ses parois de verdure, le plus souvent taillées à la verticale, devait généralement border les nombreuses percées. Pour répondre au style de l'époque, cette masse de végétation ligneuse devait être "régulière ) de forme, de consistance et de teinte - suffisamment touffue sur toute sa hauteur, du sol à l'extrémité de la cime des arbres, pour ne pas laisser entrevoir de vides trop sensibles. 
Dans les parcs paysagers, au contraire, mis à la mode au siècle suivant avec le goût de la nature, une violente réaction s'était produite contre tout ce qui offrait un caractère conventionnel. Il fallait qu'en dehors des allées devenues sinueuses, trop étroites pour les foules, l'usager puisse trouver sur les pelouses et sous le couvert des bois cet espace de verdure qui, sans apprêt apparent, lui donnerait l'illusion de la campagne, avec son calme reposant et son charme pittoresque, source de réconfort dans l'oubli momentané des soucis et des fatigues de la vie quotidienne.

De nos jours, c'est bien ce sentiment qui, de plus en plus, attire nombreux les gens de tout âge et de toute condition dans les bois proches des grandes agglomérations urbaines. Sans doute, chacun y recherche la saine distraction qui répondra plus particulièrement à ses préférences: pour les uns, l'exercice physique un peu :violent, les jeux, le sport, le scoutisme; pour d'autres, la promenade, la lecture, le stationnement au grand air, mais aucun ne restera insensible au décor naturel qui l'entoure et qui doit rester d'aspect agréable, sans pouvoir être jamais altéré par quelque fâcheux enlaidissement.

Ce n'est pas le promeneur qui, sauf cas de nécessité absolue, doit s'adapter à un cađre qui ne lui plairait pas. C'est le cadre qui doit ctre adapté aux goûts et sentiments du promeneur. " Les promenades sont faites pour le promeneur ), proclamait très justement autrefois M. l'Inspecteur Général des Eaux et Forêts Demorlaine. Il ne faut pas que ce soit l'inverse. Telle est la considération, à première vue naive, mais essentielle, qui doit guider la recherche du traitement à appliquer aux massifs boisés des parcs et des promenades. Le revenu à tirer du produit forestier est négligeable par rapport au plaisir et à l'agrément, dont la valeur ne se chiffre pas en monnaie, qu'une promenade peut et doit procurer aux nombreux risiteurs qui fréquentent les bois.

Pour qu'un massif boisé soit agréable au promeneur, il faut que, sur toute son étendue, le peuplement reste toujours suffisamment complet, ombragé, sans grands vides apparents, afin que l'intervention, trop souvent brutale de l'homme ne se laisse pas entrevoir. Il faut qu'en permanence et sur la totalité du massif puisse être assurée la continuité, non seulement de l'état hoisé, mais encore d'un colivert suffisant.

Cette règle primordiale qui trouvera son application aussi bien lans le cadre d'un parc régulier que dans les promenades à sousbois fréquentés, doit être le point de départ qu'il ne faut jamais perdre de vue, lorsqu'on étudie le traitement et le mode de régénération à adopter dans la conduite des peuplements boisés de parcs et de promenades. Sans doute, loin de nous l'idée qu'il faille appliquer cette directive, indistinctement à de vastes massifs domaniaux, plus 
ou moins visités par les promeneurs du dimanche et des congés payés ou dont quelques cantons restreints sont seuls fréquentés par des promeneurs, lorsque ces massifs procurent en matière et en argent une production que l'on aurait bien tort de restreindre. Mais cette directive s'impose, sans conteste, à tous les massifs qui ont avant tout une destination de promenade, qu'ils la tiennent de dispositions légales (Bois de Boulogne - Bois de Vincennes) ou des circonstances (Parcs de Sceaux - Ancien parc Hachette, à Robinson) (pour ne parler que des propriétés du Département de la Seine). Et elle s'impose de même avec tous les parcs et promenades, publics ou privés, faits avant tout pour l'agrément, bien plus que pour un rendement matière.

Que, dans un massif de parc ou de promenade, il soit nécessaire de maintenir la continuité du couvert, chacun le ressent d'instinct, aussi bien l'usager, plus ou moins averti en matière de sylviculture, qui supportera mal et désapprouvera la suppression d'un arbre familier, que l'architecte paysagiste, le jardinier en chef ou le Conservateur de la promenade, trop portés à ajourner tout abattage le plus longtemps possible. Cet esprit de conservation poussé à l'extrême s'est révélé fâcheux de conséquences, aussi bien pour certains de nos grands parcs nationaux (Versailles, entre autres) que pour les deux principales promenades de Paris: Boulogne-Vincennes. C'est qu'en effet l'arbre n'est pas un matériau inerte comme la pierre utilisée par l'architecte de bâtiment. Etre vivant et soumis aux lois de la concurrence vitale, l'arbre nait, grandit, se reproduit; il peut être plus ou moins longévif, mais il n'échappera pas à la longue à la décrépitude et à la mort, si toutefois, périssable à tout âge, il n'est pas venu à disparaitre plus tôt de maladie ou cl'accident.

Les deux lois qui, sous le second empire, cédèrent à la Ville de Paris, avec l'obligation d'en faire des promenades publiques, les deux forêts domaniales d'origine naturelle: Bois de Boulogne, Bois de Vincennes, alors aménagées en taillis-sous-futaie à la révolution de 30 années, avaient prescrit que ces deux domaines seraient distraits du régime forestier. Dès lors, on renonça à toute exploitation régulière; même les coupes d'amélioration, pourtant nécessaires à l'hygiène et au bon développement du peuplement furent suspendues. Alphand auquel Paris doit la création de la plupart de ses promenades, n'était pas un forestier, mais un Ingénieur des Ponts et Chaussées, d'ailleurs remarquable. C'était en tout cas un grand ami des arbres. En transformant en promenade le Bois de Boulogne, puis le Bois de Vincennes, il eut le très louable souci de conserver aussi intacte que possible la forêt primitive. Il évita donc de sacrifier des arbres; les voies nouvelles qu'il fit percer dans. le massif serpentaient, en harmonie d'ailleurs avec le style paysager, pour contourner les sujets qui méritaient d'être conservés. Il enri- 
chit encore les massifs forestiers, en faisant planter les vides, en même temps qu'il garnissait aussi de plants les anciens chemins en ligne droite qui n'avaient plus leur raison d'être. Mais, après que cela fut achevé, les massifs boisés furent laissés à l'abandon durant de longues années. AlphaND et ses successeurs semblèrent avoir ignoré le danger de.dépérissement qui guette un peuplement entièrement négligé, surtout lorsqu'on se trouve en station ingrate. Ce dépérissement devait être d'autant plus grave et plus rapide au Bois de Boulogne, reposant sur un sol d'alluvion sec, pauvre et filtrant, que les arbres de réserve de l'ancien taillis-sous-futaie, précieusement conservés, n'étaient pas issus de semis ou de plantations, mais de rejets de souche des arbres exploités après l'occupation alliée de $\mathrm{I} \mathrm{I} 5$, à la suite de laquelle presque tout le bois de Boulogne dut être recépé à blanc étoc. Par la stiite, le mal s'accéléra. L'affection touchante de la population parisienne pour ses arbres se traduisait par des protestations indignées, chaque fois que l'on tentait rle porter la hache clans les bois; on s'en abstenait donc, et ceux-ci en fait s'éclaircissaient de plus en plus par la décrépitude et la mort des sujets, dues à l'âge et au défaut de coupes d'amélioration faites en temps voulu. Pas de régénération. Les arbres morts étaient même laissé sur pied, tant qu'ils n'apparaissaient pas encore trop dangereux pour les promeneurs. Après I920, survint la maladie de l'orme; les vides s'aggravèrent encore; cette essence qui entrait pour une part appréciable dans la constitution des massifs boisés fait maintenant presque entièrement défaut au bois de Boulogne, tandis qu'au bois de Vincennes des trouées apparaissent de plus en plus nombreuses, marquant les emplacements où ont dî̀ être sacrifiés les sujets trop malades.

M. l'Inspecteur Général des Eaux et Forêts Demorlaine fut le premier à réagir contre cette politique de l'abandon, qui entraînait fatalement la disparition du peuplement forestier. Tout esprit, un peu averti en la matière, pouvait déjà prévoir, il y a trente ans que, faute d'intervention opportune, les vastes parties boisées du Bois de Boulogne et du Bois de Vincennes se transformeraient rapidement ct infailliblement, surtout dans le premier, en une steppe herbacée su un maquis de ronces et d'épines, où seuls les quelques bouquets de résineux plantés il y a une centaine d'années viendraient encore apporter un peu d'ombre, cle fraîcheur et de beauté. Ce n'est qu'à partir de I927 que le Conservateur en Chef des Promenades, bravant l'opinion publique, luttant contre les réactions de la presse. du Conseil Municipal de Paris, du Conseil Général de la Seine, se clécida à faire purger peu à peu les deux bois de tous les arbres morts ou mourants qui les encombraient. On est allé de ci, de là, au plus pressé, sans aucun aménagement ni règlement d'exploitation. $\mathrm{Au}$ Bois de Boulogne, par exemple, sur les 368 ha de forêt que renferme la promenade, on réalisa en I4 années, de 1927 à i940: 
I7 I.743 arbres de plus de Io centimètres de diamètre à hauteur d'homme, cubant environ 65200 mètres cubes, soit en moyenne I77 mètres cubes par hectare, c'est-à-dire plus de la moitié du volume total du peuplement.

Mieux que de longs propos, un tel exemple souligne les graves inconvénients d'une politique forestière de conservation à tout prix, d'abandon à eux-mêmes des peuplements boisés des parcs et des promenades, et la nécessité d'une réglementation qui prescrive en temps utile les abattages, répartis toutefois avec prudence, pour que le couvert reste suffisant, que sa continuité ne soit jamais fortement interrompue.

- Cette réglementation doit d'abord déterminer le régime et le mode de traitement à appliquer. Le régime à choisir est celui de la futaie. La futaie met mieux les arbres en valeur, avec leur plein développement ; elle satisfait davantage le sentiment du beau, et procure l'ombrage fourni et suffisamment haut pour faciliter sous le couvert la promenade, le sport et le repos. Taillis-sous-futaie et plus encore taillis simple sont peu indiqués. Sans doute, en conditions normales, ces régimes présentent-ils l'avantage d'assurer la continuité de l'état boisé, mais en forêt de promenade très fréquentée, ce ne serait nullement certain, à moins qu'on n'interdise au parcours les jeunes coupes, car les jeunes rejets risquent fort d'y être mutilés par les passants et par les jeux. A chaque exploitation de taillis, la continuité du couvert serait interrompue pendant plusieurs années, soit en totalité, soit en partie. Bien que ne devant être que temporaire, la dénudation partielle ou totale de la coupe, succédant à la densité d'un taillis âgé serait aux yeux de l'usager un acte de dévastation, une création de vide important, peu conciliables avec l'esthétique d'une promenade. Enfin, le parcours sous bois se heurterait fréquemment aux brins de taillis qui seraient rampants, fortement obliques ou bas branchus.

C'est donc bien le régime de futaie qu'il faut prendre. Or, là encore, la nécessité de maintenir la continuité du couvert se concilierait mal avec une exploitation de peuplement, aboutissant par une régénération successive de parcelle en parcelle à la formation d'une futaie à peu près équienne sur toute l'étendue de la parcelle. Sans doute, ces hautes futaies régulières et complètes de chêne et mieux encore de hêtre, comme de certains résineux sont, par leurs fûts clroits et élancés qui rappellent les majestueuses colonnes de nos cathédrales, une obtention magnifique que l'on peut considérer comme le tábleau le plus grandiose et le plus émouvant que puisse offrir la forêt française. Mais viennent plus tard les coupes secondaires, ne laisșant que des porte-graines isolés, suivies de coupe définitive, qui, avant que gaulis et bas perchis auront $\mathrm{pu}$ reconstituer un cou- 
vert suffisant, donnera encore à cette partie de la promenade, durant de trop longues années, un aspect de désolation, puis de nudité qu'il faut à tout prix éviter. Dans les forêts de promenade, proches de grandes agglomérations et dont la surface est trop restreinte par rapport à l'affluence des promeneurs, on ne pourrait donc songer, et c'est grand dommage, à créer sur une étendue suffisante pour les ręndre imposantes, ces magnifiques futaies régulières.

Force est donc, pour les promenades publiques, aussi bien que pour les parcs, de recourir à l'exploitation d'arbres, qui enlèvera, de ci, de là, des pieds disséminés sur une étendue de certaine importance. On en arrivera ainsi à la formation d'une futaie irrégulière, d'une futaie jardinée, où le sol ne se trouvera jamais découvert que sur des emplacements restreints; où les arbres d'âges mélangés résisteront mieux au vent, à l'incendie, aux invasions d'insectes; où toutes les couches de l'atmosphère depuis le sol jusqu'à la cime des plus hauts arbres pourront être utilisées pour la croissance du peuplement; où la continuité du couvert ne sera jamais interrompue, ou seulement légèrement, dans toute la totalité du massif, et sans qu'apparaissent de ces grands vides qui rompent l'harmonie et le charme d'une promenade au bois.

Toutefois, il ne faut pas se dissimuler qu'une telle méthode séduisante en principe devient parfois délicate dans son application, lorsqu'on n'a pas uniquement affaire à des essences d'ombre (hêtre, sapin) ou de demi-ombre (frêne, érable, charme, tilleul) et qu'on ne-veut pas évincer les essences de pleine lumière formant autrefois l'essence principale du peuplement naturel (chêne, par exemple, ou celles qui ont pu être introduites (pin).

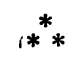

$\longrightarrow$ Lors de la réunion à Paris, en I937, des Etats Généraux de la Forêt, on avait déjà abouti à la conclusion que la régénération de promenades très fréquentées ne pouvait être obtenue qu'artificiellement, sous forme de reboisement par petits îlots de surface réduite, en des points judicieusement choisis, noyés de place en place dans les restes du massif et entourés d'une clôture en interdisant l'accès au public. Echelonnant cette restauration du massif sur un grand nombre d'exercices, M. l'Inspecteur Général des Eaux et Forêts DEMORLAINe donnait à cette méthode qu'il connaissait bien pour l'avoir appliquée depuis une dizaine d'années déjà aux bois de Boulogne et de Vincennes, après l'avoir préconisée, dès 1926, au Congrès forestier international de Rome, le nom de " Jardinage artificiel ) et la recommandait spécialement " pour la régénération des forêts de promenade autour des grandes villes, en particulier pour celles dont l'origine est un taillis-sous-futaie vieilli ». Enfin, il complétait cette méthode par deux autres opérations : éclaircie prudente, 
à intervalles rapprochés ( 5 ans au maximum), des massifs encore en bon état de végétation, mais " où l'abandon des arbres côte à côte peut faire craindre un dépérissement prochain du peuplement et introduction de hautes tiges tuteurées d'essences de valeur et longévives dans les vides de très faible étendue.

Son expérience lui avait permis de conclure qu'en raison de la trop grande fréquentation des promenades par un trop grand nombre de personnes et du piétinement du sol qui en résultait, la régénération naturelle des peuplements boisés de promenades ne pouvait être que. l'exception. Nous partageons cet avis. Le tassement du sol nuit à l'aération et à la bonne formation de l'humus. Aussi, en dehors des enclos, ne trouve-t-on aux bois de Boulogne et de Vincennes d'autres semis naturels que quelques frênes et érables, éparpillés, venus à la faveur des ronciers qui les ont protégés contre le piétinement, le plus souvent tordus pour avoir eu à lutter contre ce couvert, et finissant par être coupés ou mutilés, à la dimension de baguette ou de petite gaule par quelque promencur insouciant ou quelque enfant.

Les mêmes avatars sont survenus aux jeunes plants de hêtre qui en 193I et 1932' avaient été introduits en -sous-étage, sur I 50 hectares, dans les massifs les plus clairs du bois de Boulogne. Piétinés, mutilés par les promeneurs, ces plants ont presque tous disparu, et déjà, en 1940 , on ne retrouvait plus que fort rarement un pied de hêtre provenant de cette tentative que des clôtures n'avaient pas protégée.

Quant aux plantations de Hautes tiges, elles ont permis de combler certains vides peu importants du Bois de Vincennes, malgré qu'un certain nombre de ces hautes tiges ait été mutilé à l'époque des restrictions de chauffage. Les chercheurs de bois enlevaient les tuteurs; les tiges ne tardaient pas ensuite à se trouver brisées ou abîmées.

Au bois de Boulogne, dès I940, ces plantations de haute tige ont subi un insuccès presque total. Dans cette promenade à sol ingrat, les premières plantations faites sans apport de terre végétale n'avaient pas tardé à dépérir puis à stuccomber. Les suivantes, faites avec apport de terre végétale reprirent convenablement, mais ne résistèrent pas longtemps au vandalisme. Non seulement les tuteurs furent volés, comme au bois de Vincennes, mais encore les délinquants, profitant de l'insuffisance de surveillance des gardes de la Préfecture de Police, coupèrent rẹz terre avec de petites scies de poche les tiges introduites, même celles qui mesuraient déjà 8 centimètres de diamètre. Ce n'est qu'aux abords des voies de communication les plus fréquentées où les vandales hésitaient à opérer, que l'on peut encore retrouver quelques-unes de ces hautes tiges feuillues ou résineuses en état satisfaisant de végétation.

Malgré ces échecs, d'ailleurs localisés, la plantation de Hautes 
tiges, préconịsée par M. l'Inspecteur Général des Eaux et Forêts Demorlaine ne mérite pas d'être systématiquement abandonnée. Elle trouvera toujouris son emploi, là où les délits sont moins à craindre, notamment dans les parcs publics et mieux encore dans les propriétés privées. C'est qu'en effet, cette opération présente l'intérêt de combler de suite des vides restreints. Dans les parcs réguliers à la Française, elle permet de renforcer les parois verticales de verdure ou de boucher immédiatement une trouée sitôt qu'un arbre vient à faire défaut.

Les. éclaircies préconisées dans les portions de massifs encore en bon état de végétation, mais où l'abondance des arbres côte à côte pouvait faire craindre un dépérissement prochain du peuplement ne reçurent avant la dernière guerre qu'un commencement d'exécution. Les 368 hectares de forêt que renferme en fait le Bois de Boulogne furent répartis en 5 Divisions d'à peu près égale importance et qui devaient être parcourues de proche en proche, à raison d'une division par saison d'hiver. Ce programme dont l'exécution devait débuter.pendant l'hiver I933-I934 aurait dû s'achever avant le printemps I938. En fait, faute de main-d'œuvre ou de crédits, il ne put être appliqué. On donna logiquement la priorité à l'extraction des chablis et arbres morts sur la totalité du massif. On ne parcourut que 50 hectares, en coupes d'amélioration très modérées, sans asseoir ces coupes de proche en proche. On allait de ci, de là, sans ordre préalable, en courant à ce qui paraissait le plus urgent.

On ne trouve pas qu'au Bois de Vincennes, un programme d'éclaircie aussi strict ait été établi. Les éclaircies s'y poursuivirent cependant, sans interruption, de I934 à I940, en sus de l'exploitation des chablis. 92 hectares furent parcourus sur les 330 ha de peuplements forestiers que cette promenade comporte.

Lorsque survinrent la guerre, l'occupation ennemie, les pénuries de toute sorte, un gros effort fut, dès la fin de l'année I940, réclamé des bois de Boulogne et de Vincennes. Il fallut sur place constituer un important stock de précaution de bois de chauffage pour les boulangeries de l'agglomération parisienne, pourvoir à la fabrication de charbon carburant nécessaire aux voitures à' gazogène de la Ville de Paris et des Pompes Funèbres. A cette époque, bien que purgé depuis I92'7 de ses arbres morts et des plus dépérissants, le bois de Boulogne n'offrait certes pas belle apparence à l'œil du. forestier quelque peu averti et demeurait encore encombré d'arbres tarés. Le fond du peuplement était formé de chênes, réserves de l'ancien taillis-sous-futaie, issues de rejets d'arbres et perches de taillis vieilli (généralement rouvrẹ et pubẹscent dans la moitié Nord, pédonculé 
dans la moitié Sud). Les unes et les autres n'ayant été l'objet d'aucun soin ni coupes d'amélioration opérées en temps utile, laissaient pour la plupart entrevoir à leurs cimes des moignons de branches mortes, présageant la fin prochaine de l'arbre. A côté, les vieux acacias, essence introduite et devenue envahissante, se trouvaient atteints de pourriture au, pied et leur ramure, en partie sèche, devenait pour le promeneur un danger permanent. Beaucoup d'érables sycomores étaient aussi pourris au pied; le fût de la plupart des peupliers sonnait creux. L'orme avait disparu; quelques autres essences disséminées: bouleau, parvenant à l'âge de la décrépitude, frêne, tilleul, charme dont la cime étalée masquait trompeusement la pauvreté du peuplement, complétaient les massifs feuillus. A l'exception des bouquets résineux, le peuplement était donc en fort mauvais état.

Quant aux trouées, ouvertes pour être reboisées et qui, en principe, n'auraient pas dû dépasser chacune la surface de 50 ares ( $\mathrm{I}$ ha tout au plus, avait-il été concédé), elles se trouvaient au nombre de 56 , clôturées, occupant une surface de 20 ha 36 ares et l'on y avait introduit 74095 plants repiqués âgés de 3 à 8 ans, avec quelques hautes tiges en remplacement des manquants. Mais, si au début l'ouverture de ces trouées stivies de plantations avait été faite très timidement, sur quelques ares tout au plus, il n'en fut pas longtemps ainsi. Dès que la réaction du public commença à se calmer devant le succès des premiers essais, la surface de l'enclos de reboisement fut sensiblement agrandie, soit par raison d'économie, soit pour faciliter la reprise des plants; le plus grand de ces enclos atteignit une surface de 7 ha 50 ares. La limite imposée avait donc été largement dépassée. Afin de masquer un peu l'importante rupture de continuité ainsi apportée au couvert de la promenade, on avait bien, au début, pris la précaution de maintenir à l'intérieur de ces trop grands enclos quelques arbres de l'ancien massif semblant avoir encore une vitalité suffisante, mais bien qu'on eût pris la précaution d'en relever le couvert en élaguant leurs basses branches, ces réserves s'étaient montrées plutôt préjudiciables aux plants d'essences de lumière malencontreusement introduits sous leur ombrage, et il avait fallu les exploiter. Il en était donc résulté dans la promenade des vides beaucoup trop importants qui devaient attendre un certain nombre d'années avant d'être masqués par les jeunes plants ayant atteint une hauteur suffisante, puis, lorsque ce résultat commençait à être acquis, l'incendie ravagea deux de ces importants enclos, le sinistre ne put être maitrisé à temps, le vide réapparut encore pour longtemps et il a fallu reprendre le reboisement.

Le bois de Vincennes repose sur un sol plus frais et plus fertile dans son ensemble que le Bois de Boulogne. Aussi porte-t-il des arbres plus vigoureux, plus élancés, et les vides, dans le peuplement, y sont-ils moins importants. Le peuplement provient encore d'un an- 
cien taillis-sous-futaie de chêne où toute exploitation régulière avait été suspendue, il y a 90 ans, dès l'acquisition de ce domaine par la Ville de Paris. En respectant et laissant vieillir les cépées de taillis, sans toucher aux arbres de réserve, on espérait aboutir à la formation d'une futaie complète, mais, bien que de franc pied pour certains, beaucoup de chênes s'étiolent, dépérissent, meurent peu à peu. Les ormes plantés dans la seconde moitié $\mathrm{du} \mathrm{XIX}^{0}$ siècle succombaient depuis 1930, l'un après l'autre; les acacias, les peupliers et quelques autres essences disséminées: érables, tilleuls, frênes introduits il y a une centaine d'années, arrivaient à terme. On peut noter la présence de quelques bouquets de résineux, presque tous pins: laricio noir, sylvestre. Dans l'ensemble, l'état du peuplement en 194I paraissait moins mauvais qu'au bois de Boulogne, mais, là aussi, on n'avait pu obtenir de régénération naturelle sur laquelle on puisse compter. A cette époque, 88 enclos de reboisement avaient été clôturés, occupant une surface de 22 hectares, et l'on y avait introduit 50434 plants repiqués, âgés de 3 à 8 ans; en dehors des clồtures, on avait planté 5.28I hautes tiges. Les trouées avaient été ouvertes avec modération, suivant les directives prescrites à l'origine; le plus grand enclos était de 62 ares. La plupart des arbres laissés dans les enclos au moment de leur création avaient pu être maintenus; les réserves avaient plus d'avenir qu'au Bois de Boulogne et les plants introduits sous leur couvert avaient été judicieusement choisis parmi des essences d'ombre. La promenade ne fut donc jamais entr'ouverte comme au. Bois de Boulogne de vides importants rompant la continuité du massif.

- Forts de ces précédents, il devenait alors possible en I94I, en vue d'asseoir convenablement les fortes exploitations nécessitées par les circonstances, de dégager et fixer avec précision la doctrine qui serait pour les deux promenades la base de l'aménagement forestier à suivre. Il fallait à tout prix fournir du bois; il eut été fâcheux de continuer à courir de ci, de là, à travers les massifs; au plus pressé des peuplements dépérissants, sans ordonner au préalable l'assiette des coupes.

Nous avons vu que le traitement en forêt jardinée s'imposait. C'est celui qui a été alors prescrit officiellement dans le Bois de Boulogne et dans le Bois de Vincennes. Le jardinage dont il s'agit est un jardinage par parquets, par bouquets équiennes dont l'étendue et le contour seront très variables; ce n'est pas un jardinage pied par pied qui aurait abouti à la longue à éliminer les essences de lumière. L'assiette des coupes imposées était une assiette par contenance. suivant un ordre établi par le règlement d'exploitation. Cependant, comme dans ces peuplements de promenades très fréquen- 


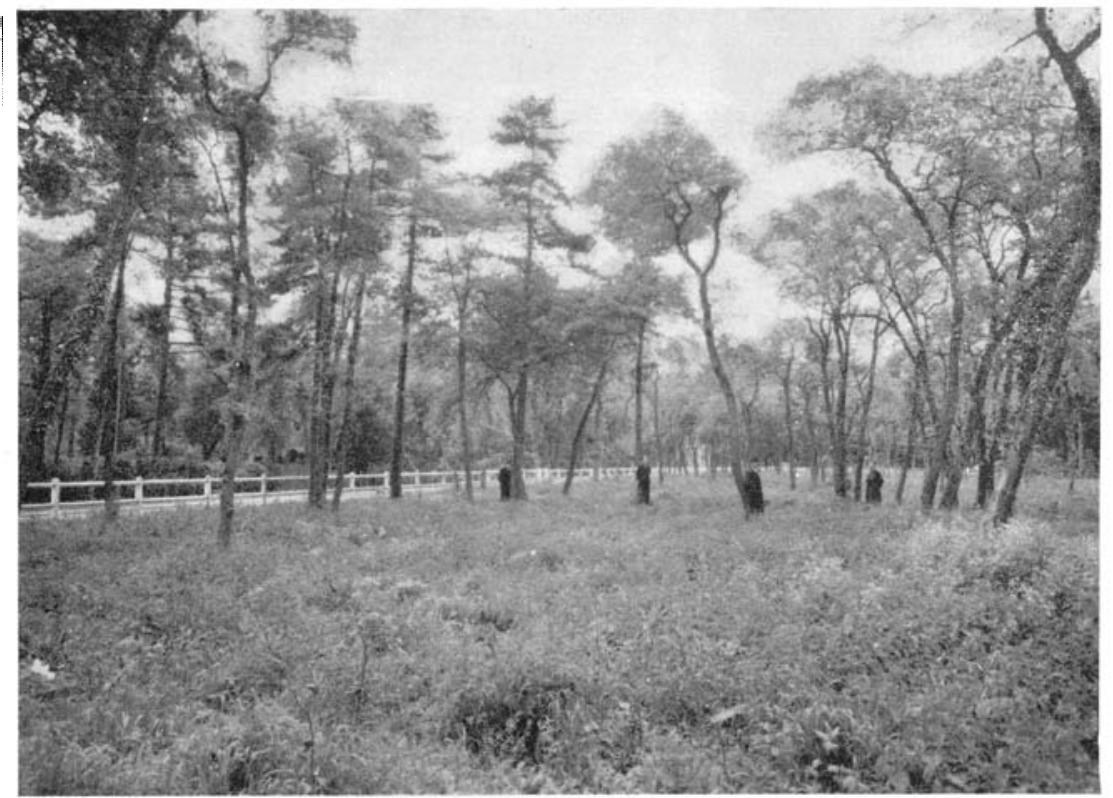

Bois de Boulogne (Enclos 66). Devant Bagatelle.

Plantation de hêtre âgée de I an le 5 mai 1944.

(Cliché Service de la Préfecture de la Seine.)

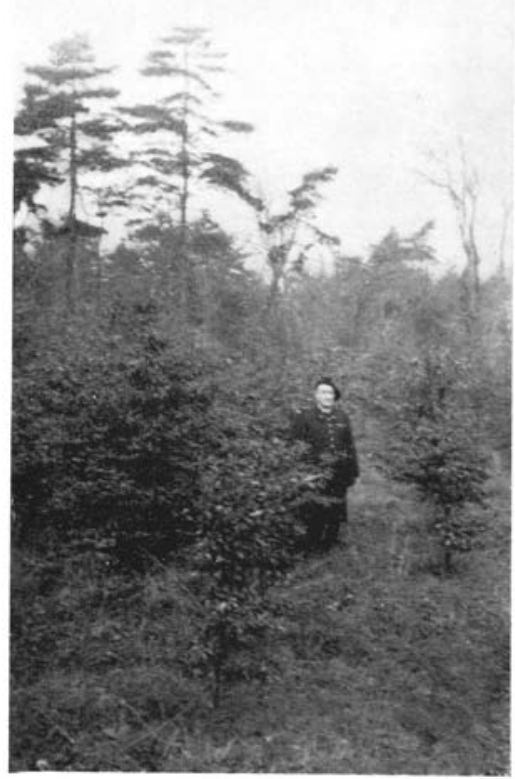

Même plantation le 5 mars 1955 . Hêtres de 12 ans.

(Cliché Toussaint.) 


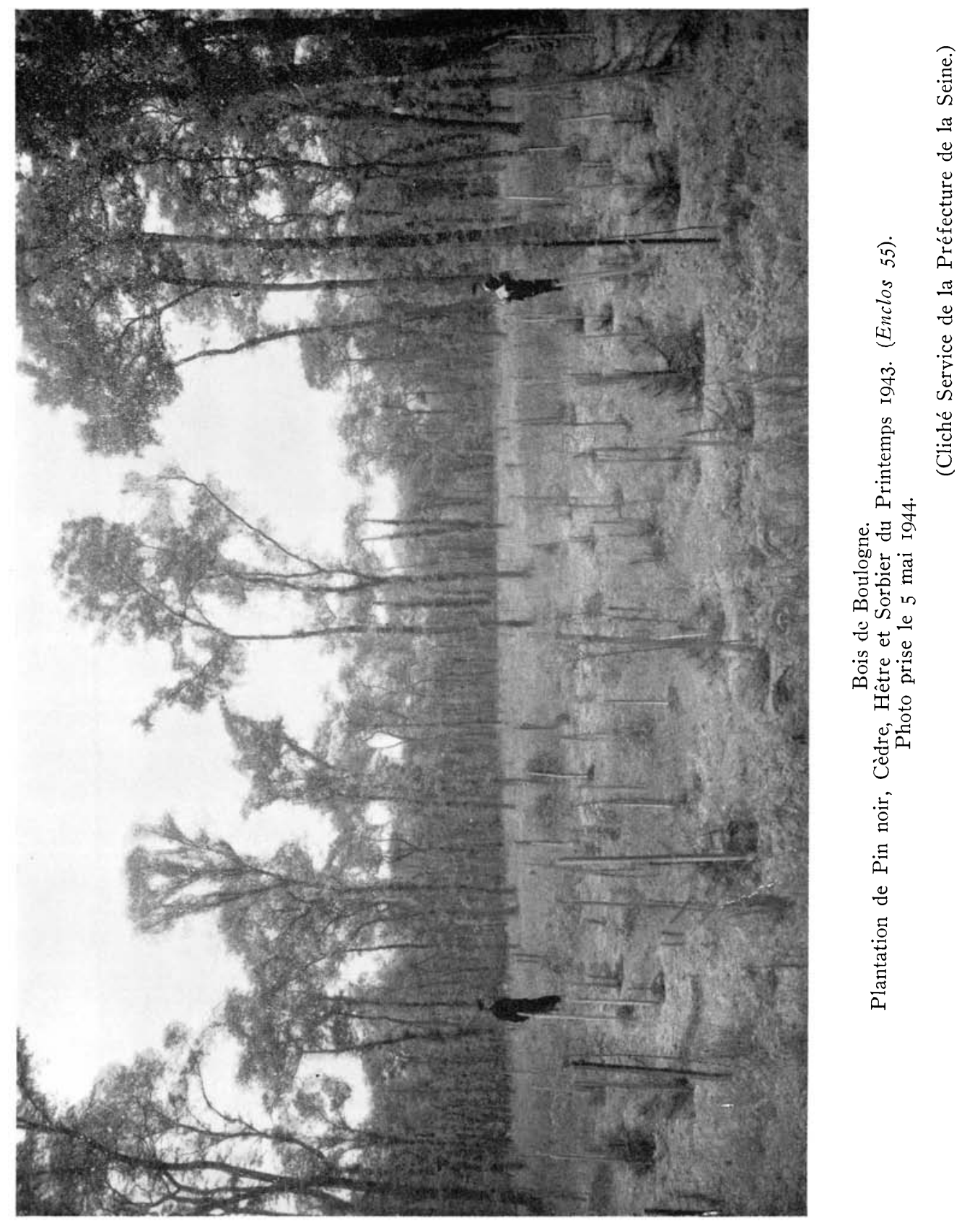




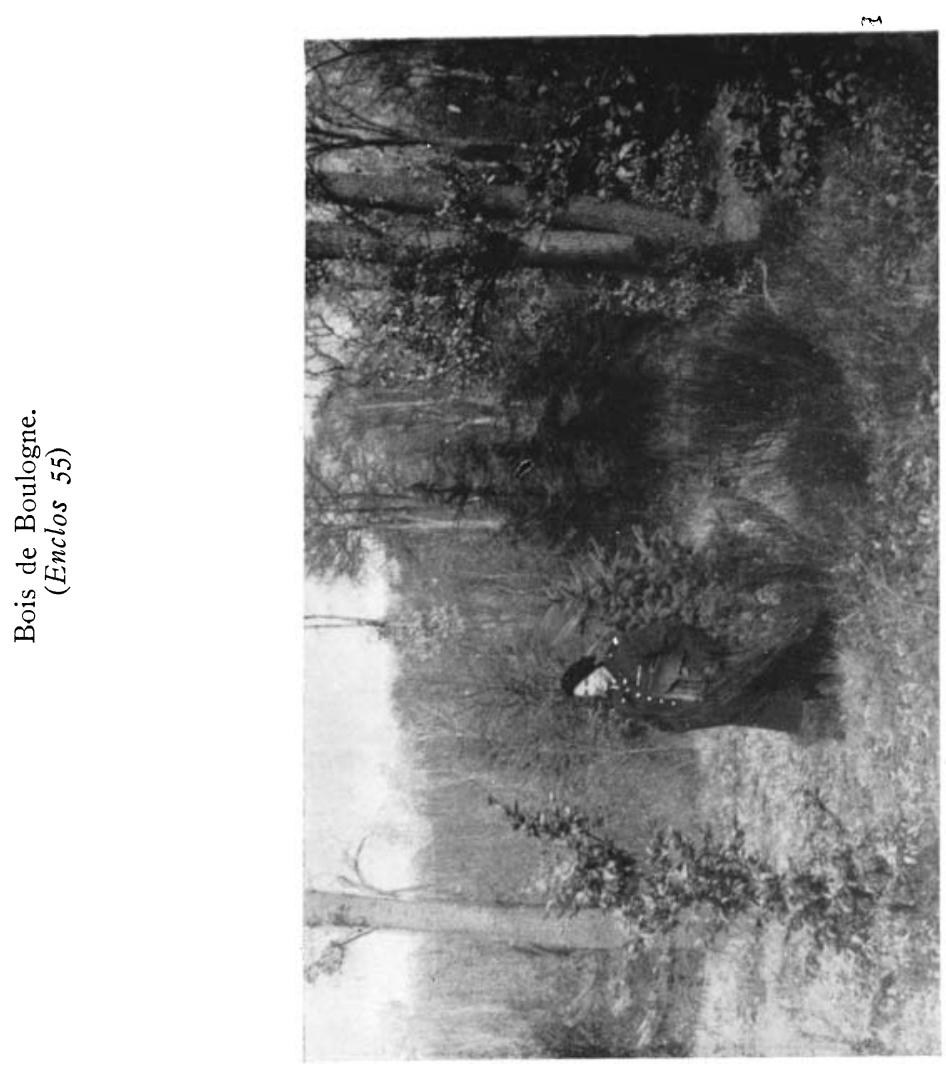

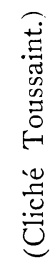

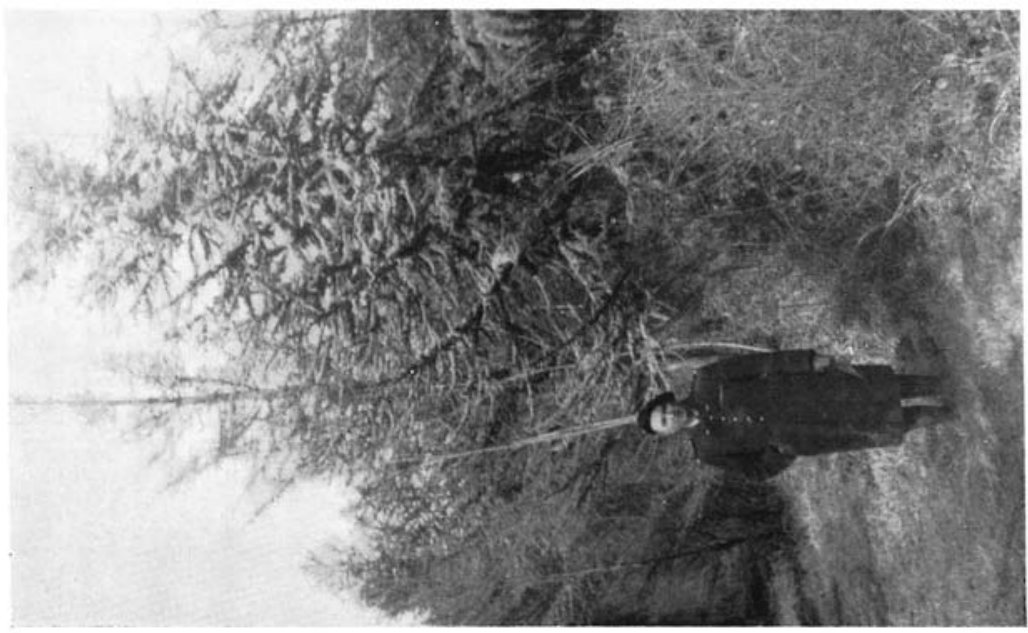




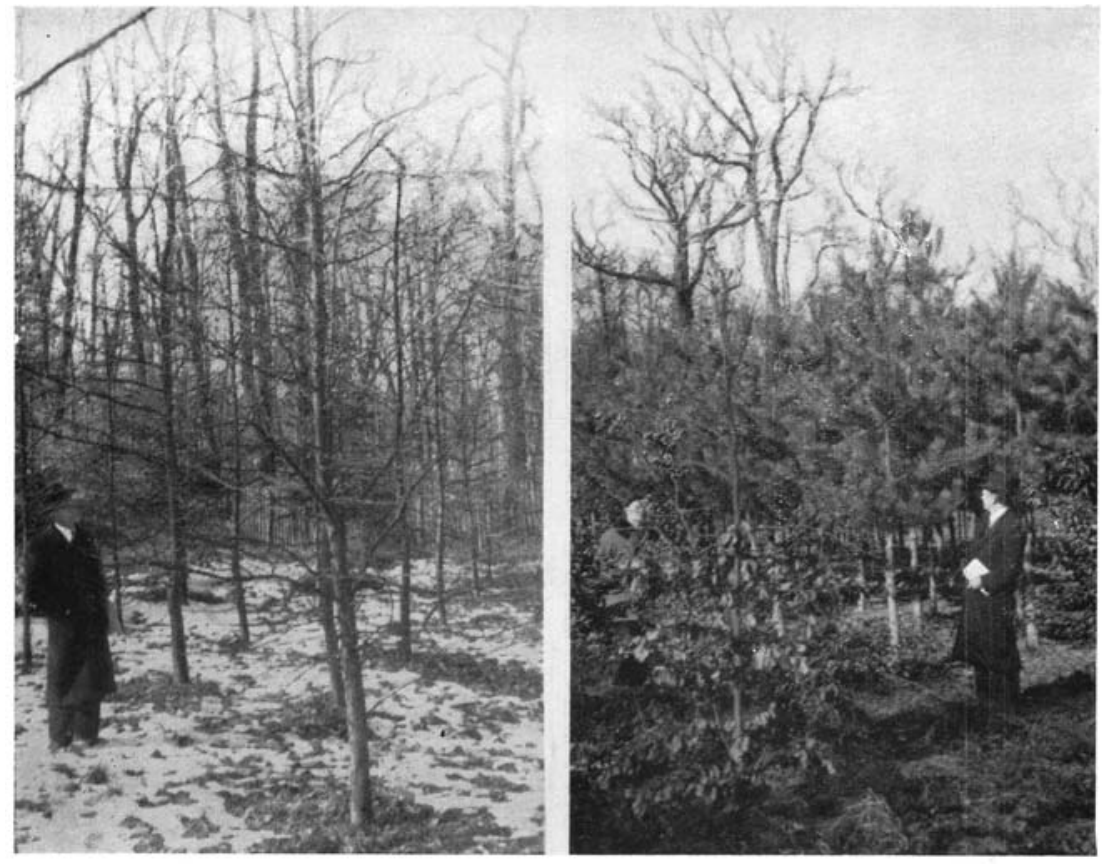

Bois de Vincennes.

Enclos 69

Liquidambar I3 ans de plantation.

Enclos $I$

Vide de o ha 34 creusé par une bombe le jour de la Libération de Paris.

Pins noirs et Hêtre

en Croix de Lorraine.

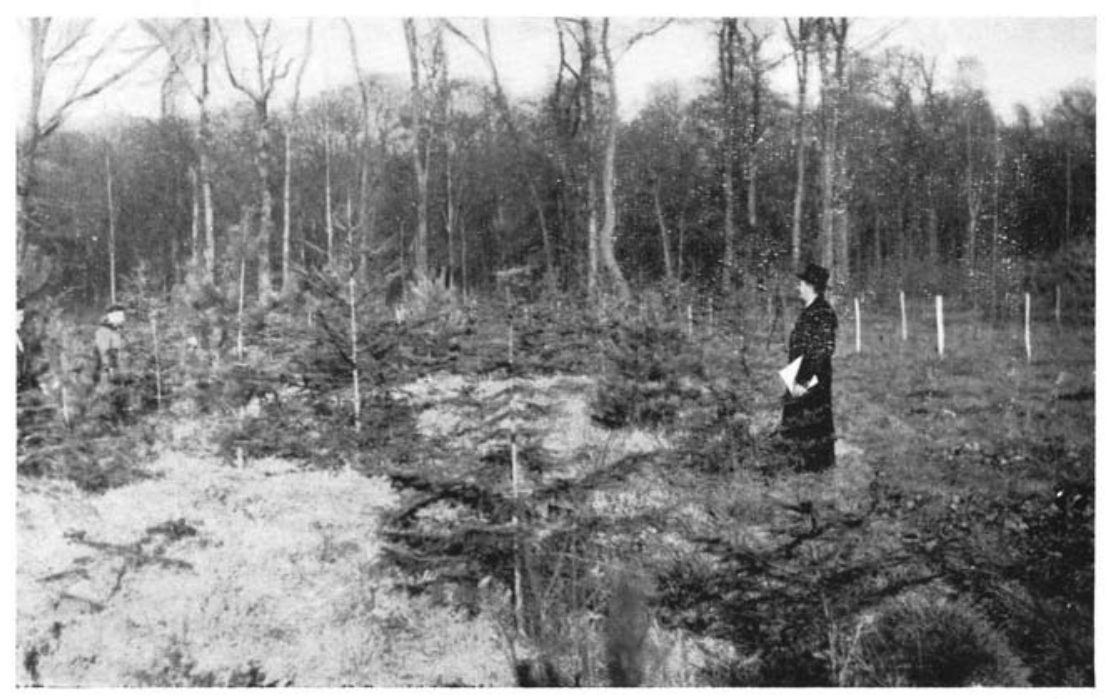

Bois de Vincennes.

Enclos 32

Plantation de 8 ans de Pin noir, Douglas et Nordmanr.

(Clichés Toussaint.) 
tés, arrivant peu à peu au terme de leur existence, il est très utile de se rendre compte du natériel restant et d'en suivre les fluctuations, non seulement les arbres marqués en abandon, mais encore tous ceux qui étaient réservés devaient être comptés, mesurés et cubés, en utilisant pour le cubage un tarif uniforme, spécial pour chacune des promenades (les arbres du Bois de Vincennes ayant à diamètre égal plus de hauteur que ceux du Bois de Boulogne). Le contrôle, ainsi prescrit, devait permettre au " Maitre de la forêt ) de se rendre compte de l'enrichissement ou de l'appauvrissement des massifs boisés et le guidait dans ses opérations de coupe et de régénération artificielle. Mais, pour que ce contrôle puisse être opérant, i' faut que le comptage soit refait à intervalles réguliers et qu'il puisse être comparable d'une année à l'autre. Le tarif d'origine, quelque fictif qu'il puisse être, s'il a été mal établi, s'impose donc toujours obligatoirement; les arbres doivent toujours être mesurés au même niveau, indiqué sur l'écorce par un coup de griffe horizontal, superficiel, qu'on rafraîchira à chaque comptage.

- Dans les massifs boisés des parcs et jardins, comme dans les forêts affectées à la promenade, telles Boulogne et Vincennes, la coupe jardinatoire doit être guidée par des considérations à la fois culturales et esthétiques, ainsi que nous l'avons vu. Là nì le peuplement possède un état de végétation encore assez satisfaisant et se montre encore suffisamment dense, une éclaircie modérée s'impose, à intervalles assez rapprochés dans son renouvellement. Cette opération doit bien se garder d'occasionner des trouées visibles dans le peuplement qui doit demeurer suffisamment fermé pour qu'un observateur non averti passant peu après dans la coupe ne puisse se rendre compte par le seul aspect du couvert, qu'à cet emplacement le peuplement vient d'être l'objet d'une exploitation.

Cette coupe d'amélioration doit naturellement favoriser l'essence précieuse, mais dans une promenade le prix commercial du bois n'est pas le facteur primordial qui donne de la valeur à un arbre. Il va de soi que des essences ayant un bois de valeur doivent, si elles peuvent atteindre un certain âge, tels le chêne, le hêtre, le frêne, être considérées comme précieuses dans un peuplement boisé de promenade - toutefois il est aussi d'autres arbres précieux, non par leur bois ou leur longévité, mais par leur rareté ou la valeur décorative que, suivant les circonstances, ils prendront dans l'ensemble du peuplement: un résineux, par exemple, disséminé par pieds isolés ou par petits groupes dans un massif feuillu - quelques bouleaux formant contraste avec la teinte sombre de résineux ou mêlés à des feuillus de teinte foncée - des sorbiers, des merisiers, etc... qui, à certaines saisons, apporteront une note de couleur plus vive, etc...

Tout en évitant de faire une trouée, la coupe d'amélioration réalisera, avant tout, les arbres morts; pui, les arbres tarés et dépérissants, tout au moins parmi ces derniers ceux qui risquent de périr 
avant que vienne en tour une nouvelle exploitation - enfin, les arbres laids au point de vue esthétique, lequel pourra sensiblement différer dı point de vue forestier. Un arbre mal conformé peut par sa forme pittoresque ou bizarre être un sujet d'étonnement ou d'admiration pour le promeneur ou même tenter le pinceau d'un artiste; il devient dès lors intéressant et il faut bien se garder de le faire abattre, s'il est encore sain. Quant aux arbres dominés, s'ils ne sont pas tarés et ne nuisent pas à leur voisinage, il est préférable de les conserver, même s'ils n'ont pas d'avenir apparent; ils contribuent à renforcer la densité du couvert et peuvent, à l'occasion, trouver leur utilité comme sujets de remplacement, si un accident survient à l'arbre qui les surplombe.

Quelques pas plus loin, dans la même parcelle, l'opérateur pourra rencontrer un peuplement dégradé, en fort dépérissement, et dont il peut entrevoir la disparition rapide. Ce peuplement n'est pas nécessairement honogène; à certains emplacements, on peut y voir des pieds ou des bouquets d'arbres susceptibles de vivre encore un certain temps. Alors la coupe de jardinage consistera à ouvrir de petites trouées, disséminées plus ou moins régulièrement suivant ce que l'on trouve. Tantôt la trouée est déjà bien apparente; il suffit pour cela que quelques arbres morts aient été extraits précédemment au titre cle chablis; on peut être amené à l'agrandir encore un peu, en enlevant les mauvais arbres du pourtour. Tantôt, sans être encore apparente, la trouée existe déjà en fait, masquée par des épines et des broussailles qu'on devra faire recéper ou extraire. Tantôt c'est l'opérateur qui créera la trouée en abattant tout un groupe d'arbres tarés ou surannés. La forme et les dimensions de ces trouées doivent être adaptées aux circonstances, mais il faut éviter d'en exagérer la dimension et de dépasser l'étendue de $5^{\circ}$ ares, car il importe que les trouées soient bien noyées dans le surplus du massif, afin de maintenir aux yeux des promeneurs la continuité du couvert, et, en bordure des chemins, on devra toujours s'efforcer de conserver un rideau d'arbres ou même de broussailles pour masquer le vide. Autour de la trouée, le peuplement doit être maintenu aussi compact, aussi clense que possible, pour éviter que le vent s'engouffrant dans le vide vienne à provoquer des chablis sur le pourtour.

M. l'Inspecteur Général des Eaux et Forêts Demorlaine conseillait de conserver dans les trouées à reboiser tous les arbres susceptibles de vivre encore un certain temps, en les élaguant, de façon à relever leur couvert et à permettre sous leur abri la végétation d'essences plus précieuses. Dans son esprit, ces réserves étaient utiles, les premières années, pour abriter les plants introduits contre la gelée et la sécheresse et pouvoir encore, au besoin, servir des porte-graines. Mais il prescrivait de les faire ensuite disparaitre petit à petit, au fur et à mesure du développement des essences introduites. En 
fait, l'utilité de ces réserves nous apparait surtout comme masque des trouées, en attendant que les jeunes plants se soient développés, mais si la trouée ne clépasse pas 50 ares, le maintien de ce masque est moins nécessaire. Quelle que puisse être l'habileté des bûcherons qui débitent l'arbre réservé avant de l'abattre (ce qui exigera huit fois plus de temps qu'un abattage ordinaire), la jeunesse voisine risque d'être mutilée. Sous le couvert des arbres, les jeunes plants, même d'essence d'ombre, poussent moins vite et arrivent ensuite difficilement à récupérer ce retard, lorsqu'ils sont clégagés. C'est pourquoi nous estinions préférable de ne conserver dinns une tronée que des arbres de lumière et d'essence longévive (chêne, par exemple), ayant encore une belle végétation, bien conformés et qui puissent se raccorder plus tard au nouveau peuplement que l'on crée par plantation. Et voici maintenant que l'opérateur se heurte au cours de son opération de martelage à la clôture d'un enclos précédemment reboisé. Il devra bien se garder de le négliger et le contourner, mais profiter justement clu passage de la coupe pour pénétrer dans cet enclos, y vérifier l'état des réserves, y asseoir en queque sorte la coupe secondaire si ces réserves paraissent trop alondantes, supprimer toutes celles qui lui paraitront inutiles et ne poutvoir se raccorder au peuplement nouveau, élaguer et relever le couvert des arbres qu'il juge pouvoir être définitivement maintenus. Il aura soin également d'examiner le reboisement, d'y prescrire les opérations d'entretien qui s'imposent dans la jeunesse: un vide à regarnir, des sujets d'élite à dégager, une cime à défourcher, une tige à tuteurer, etc... Rien ne sert de consentir de lourds sacrifices en argent et en travail pour faire des plantations, si par la suite on en néglige l'indispensable entretien. Il tombe sous le bon sens que si main-d'œuvre et crédits deviennent insuffisants, mieux vaut employer ce dont on dispose pour assurer le bon état et la conservation cles travaux de régénération antérieurs que d'entreprendre des travaux neufs en négligeant et risquant de perdre ce qui avait été fait antérieurement.

Le jardinage par trouées suivi de plantations ne peut donc être voir assurer la péremnité des forêts de promenade et maintenir. sans interruption dans le temps la continuité du couvert. Il ne faut pas que tous. les cent ou cent cinquante ans, on risque de voir brusquement disparaitre en même temps l'ensemble du couvert, comme cela se serait produit de nos jours si l'on n'y avait remédié à temps et comme celà s'était déjà malheureusement produit à diverses époques de l'histoire de nos promenades parisiennes: Bois de Boulogne. en 1790-I795 - en I8I6 et partiellement en I870-I87I - Bois de Vincennes, en I4I9-I 55 I-I 73 I-I 865 .

Le jardinage par trounées suivi de plantations ne peut donc être 
interrompu; la restauration doit être permanente, s'échelonnant sur une surface plus ou moins étendue suivant les circonstances, surtout suivant l'état et la rapidité de dégradation des massifs, mais elle devra aussi se concilier avec le souci d'apporter le moins de gêne possible aux usagers de la promenade.

Nous avons vu que les trouées doivent être clôturées, sauf celles qui ouvertes en des points de surveillance facile peuvent sans trop de risque être garnies d'arbres de moyenne ou de haute tige,.attachés à un tuteur et à l'occasion entourés d'un faisceau d'épines.

La formation d'enclos de petite étendue ou de contour irrégulier entraînera proportionnellement aux surfaces respectives une longueur de périmètre supérieure à celle de vastes enclos ou d'enclos à contour carré: On peut en tenir compte, par mesure d'économie, sans toutefois dépasser par enclos une surface de o ha 50 ares. Lorsque plusieurs trouées sont voisines, on peut avoir intérêt à clôturer l'ensemble, plutôt que de mettre une clôture autour de chacune, mais il ne faut pas que ce soit un motif pour sacrifier et remplacer par une nouvelle plantation les rideaux d'arbres dans lesquels sont noyées les trouées et qui contribuent à maintenir la continuité du couvert sur ce point de la promenade.

Dans des promenades très fréquentées, on ne peut interdire sur de trop grandes étendues le parcours des promeneurs. Il a été admis en I94I que la surface des enclos de reboisement ne devrait pas excéder le cinquième de la surface totale des massifs boisés.

Après entourage des enclos par une clôture et plantation de jeunes plants repiqués de 2 à 3 ans, il faut compter environ 25 années avant de pouvoir supprimer la clôture; gaulis et bas perchis obtenus pourront alors se défendre. Il faut aussi prendre soin que les clôtures gênent le moins possible les promeneurs. Il ne faut pas que le public se heurtant sous bois à un enclos ait à faire un trop long détour pour reprendre sa marche dans la direction désirée; on doit également s'efforcer de répartir judicieusement les enclos et éviter de les multiplier brusquement sur un point déterminé de la promenade. Ce sont là des sujétions parfois embarrassantes, mais dont on doit bien tenir compte lorsqu'on applique une méthode qui, faite pour concilier à la fois les nécessités culturales d'un peuplement forestier et la jouissance d'une promenade très fréquentée doit, pour aboutir au résultat cherché, être employée avec beaucoup de doigté et une certaine souplesse.

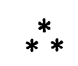

- Il a été tenu compte de ces considérations et de !'état des peuplements pour réglementer en I94I l'aménagement du Bois de Boulogne et du Bois de Vincennes.

Ce règlement a prescrit la division de chacun de ces deux massifs boisés en dix parcelles numérotées de I à Io, sans que toutefois cette 
division puisse, comme en forêt domaniale, apparaitre aux yeux des promeneurs, et, suivant l'ordre imposé par un règlement d'exploitation, l'assiette par contenance de coupes d'éclaircie très prudentes accompagnées de coupes jardinatoires, faites par petites trouées suivies immédiatement d'un reboisement clôturé. La rotation était au maximum de Io ans. C'était en somme du jardinage par parquet, tel que nous venons de l'exposer.

Chacun des deux bois fut partagé en dix parcelles délimitées naturellement par le périmètre ou des chemins et se suivant de proche en proche dans le numérotage, avec une contenance aussi équilibrée que le permettait la topographie des lieux. Chaque parcelle fut ensuite subdivisée en sous-parcelles, désignées par des lettres arabes, et séparées les unes des autres par des chemins ou des ruisseaux - ceci, pour pouvoir mieux situer les arbres les plus intéressants, les enclos de reboisement, les plantations de hautes tiges. etc... On ne mentionna ces divisions que sur les plans, sans marque visible dans la promenade.

Les exploitations du premier tour, commencées le $\mathrm{I}^{\text {er }}$ janvier I94I se trouvaient déjà achevées sur la totalité des deux bois le $\mathrm{I}^{\text {er }}$ avril I944. Elles furent assises avec le plus de modération possible, uniquement guidées par des considérations culturales. C'est ainsi que dans le Bois de Boulogne, on exploita 30260 pieds d'arbres cubant I 500 mètres cubes de gros bois, soit une moyenne de 3I mètres cubes par hectare.

Lorsque survint la libération de Paris et que l'on dut appliquer momentanément le plan d'extrême détresse, on préféra sacrifier dans les deux promenades des arbres d'avenue, tels au Bois de Boulogne les vieux acacias de l'Avenue de Longchamp, plutôt que de reprendre trop vite une nouvelle rotation dans les massifs boisés. Mais la pénurie de bois alla en s'aggravant encore à Paris, et il fallut reprendre les exploitations. Un second tour fut donc entamé dans chacune des promenades, jusqu'au 30 décembre I946. Dans le Bois de Boulogne, les cinq premières parcelles furent à nouveau parcourues, toujours en jardinage par contenance, mais avec une extrême modération; on abattit 6492 pieds d'arbres, cubant 2482 mètres cubes gros bois, soit environ I4 mètres cubes par hectare. Cette opération permit entre autres de chauffer et sauver pendant le rigoureux hivers I944-I945 les remarquables collections horticoles de serre de la Ville de Paris. Suivant les comptages faits sur l'ensemble des massifs boisés du Bois de Boulogne, il restait après ces coupes, au $I^{\text {er }}$ janvier I947, 90500 arbres cubant environ 47000 mètres cubes, ce qui répond à une densité moyenne de 127 mètres cubes à l'hectare, enclos de reboisement compris. Au Bois de Vincennes, sept parcelles furent parcourues dans le second tour.

A partir du $\mathrm{I}^{\text {er }}$ janvier 1947 , les coupes par contenance furent interrompues dans les deux bois, où l'on se contenta de réaliser 
les chablis et les arbres dangereux, ainsi que quelques arbres se trouvant sur l'emplacement de nouveaux enclos de reboisement ou d'anciens enclos dont il était opportun de dégager la jeunesse. Il serait temps maintenant de reprendre ces coupes de jardinage, là où on les avait laissées en I947, et de parcourir régulièrement, suivant le règlement d'exploitation, sinon deux parcelles, tout au moins une parcelle chaque année.

Il avait été décidé, en I94I, comme nous l'avons dit plus haut, que la surface totale des enclos de reboisement ne devait pas dépasser le cinquième de celle des massifs boisés. C'est cette proportion qui, dans le programme de I94I, a été adoptée pour le Bois de Boulogne qui a 368 hectares boisés. On ne peut donc y dépasser 74 hectares pour la surface clôturée. Actuellement, au Bois de Boulogne, il y a 93 enclos occupant une surface de 62 hectares, ce qui donne une moyenne de o ha 66 par enclos, surface exagérée due à l'importance démesurée donnée à quelques-uns. Il est maintenant possible de rendre au parcours du public les premiers reboisements opérés au cours de l'hiver I928-1929, et c'est ainsi que, comme prévu, les plantations au Bois de Boulogne pourront se poursuivre au delà de 74 hectares.

$\mathrm{Au}$ Bois de Vincennes, où les peuplements boisés occupent une surface de 330 hectares, la proportion de I2 \% de cette surface pour les plantations à clôturer; fixée en I94I et qui limitait ainsi à 40 hectares le maximum de contenance totale des enclos, vient d'être portée à I6 \%, soit: 53 hectares. Il y devient aussi possible de supprimer les clôtures des premiers reboisements. Les enclos réalisés sont aujourd'hui au nombre de Io6, couvant une surface de 37 hectares, soit en moyenne 35 ares par enclos. Là, on peut dire que la méthode du jardinage par trouées prudentes a été judicieusement et correctement appliquée, sans que la continuité du couvert boisé n'ait jamais été sensiblement interrompue depuis une trentaine d'années.

- La régénération des massifs boisés cles promenades opéréc ainsi par des procédés artificiels, à cléfaut de régénération naturelle sur laquelle on puisse compter, permet à l'opérateur de modifier la nature et la proportion des essences du peuplement antérieur. Il lui est clonc possible par le choix des essences qu'il fait pour ses plantations de modifier sensiblement la composition des massifs, de les rendre plus attrayants, mieux adaptés au rôle que doit jouer un parc ou une promenade.

Une question parfois débattue est celle de l'opportunité de plantations résineuses et de limportance à leur donner dans l'ensemble de la composition.

L'ordonnance symétrique des parcs à la française du Xvire siècle, 
le rôle d'encadrement auquel se limitent généralement les boisements de l'époque, la recherche de régularité dans la forme, la consistance, la teinte des massifs y prohibent ou limitent fortement l'emploi de résineux. Ceux-ci (if, entre autres) pourront être utilisés pour des berceaux et des cabinets de verdure, pour des labyrinthes, des bordures de rampe ou d'escalier; ils n'auront pas leur place dans le peuplement forestier.

Il n'en est pas de même, au contraire, dans les parcs paysagers où l'on recherche le pittoresque, la variété, le contraste. Lorsqu'il s'agit de promenades très fréquentées en toute saison, la présence de résineux rompt la monotonie qui ressort, durant les mois d'hiver, d'un peuplement formé exclusivement d'arbres à feuilles caduques. On peut alors constater combien sont fréquentés, au Bois de Boulogne et au Bois de Vincennes, les quelques hauts perchis et futaies de pin, et il aurait été réellement grand dommage qu'un ostracisme prononcé, il y a une centaine d'années, contre les résineux de promenade eut entre autres privé le Bois de Boulogne de cette note si pittoresque formée de nos jours par les bouquets de pin laricio plantés alors sur les berges du lac inférieur, dans le but probable de masquer la faute de topographie commise en mettant trop haut le niveau du lac.

Ce n'est donc pas seulement par pieds isolés, mais par bouquets de certaine importance que l'on doit employer le résineux, et c'est fort judicieusement que, dès l'origine des plantations en I928, une assez large place lui fut faite dans les deux grandes promenades parisiennes.

Parmi les essences feuillues, le hêtre cst particulièrement indiqué pour les massifs boisés des parcs et promenades, tant en raison de la beauté de son fût et de la teinte séduisante de son feuillage aux changements de saison que de l'épaisseur de son couvert, sous lequel le sol reste nu, sans ronces, ni herbes ni orties, bien propre au parcours et aux jeux. Essence d'ombre, le hêtre s'adapte particulièrement au traitement de jardinage par trouées suivi de plantation.

Quant au chêne qui, dans les Bois de Boulogne et de Vincennes est l'essence dominante de la forêt primitive et qu'on y retrouve avec trois espèces naturelles: pédonculé, rouvre, pubescent, il ne peut être question de l'éliminer et il faut profiter des emplacements ensoleillés et suffisamment dégagés, en sol favorable, pour l'introduire dans les enclos par plantation de jeunes sujets repiqués ou par semis provenant de glands récoltés avec soin sous de beaux rouvres.

Depuis vingt-huit années, on a introduit dans lès enclos du Bois de Boulogne et du Bois de Vincennes rog espèces différentes de jeunes plants de reboisement, dont 64 feuillus et 45 résineux. Elles ont subsisté presque toutes dans l'un ou l'autre bois, de plus ou moins 
belle venue. Quelques-unes seulement ont succombé aux hivers rigoureux: Pinu, insignis, Cupressus sempervirens, Cupressus macrocarpa, mais presque toutes les autres ont résisté, notamment. Pinus pinaster (Pin maritime, race du Maroc) qui a fort bien réussi.

Avec l'expérience maintenant acquise, on peut conseiller sans grand risque d'erreur, parmi les résineux, même en terrain ingrat: le Pin laricio (noir d'Autriche, Corse, Calabre) et le Cèdre de l'Atlas, en prenant toutefois la précaution pour celui-ci de n'utiliser que des plants en motte ou de praliner soigneusement ses racines si l'on utilise des jeunes plants à racine nue. Le sapin de Douglas a bien donné satisfaction dans sa jeunesse, mais il est un peu troublant de constater l'absence de beaux Douglas de plus de cinquante ans, alors que cette essence a été certainement introduite dans ces promenades de Paris au siècle dernier. Parmi les Abies: Abies cephalonica mérite une mention spéciale; il s'est montré le meilleur parmi les sapins méditerranéens et il y a, au Bois de Boulogne, un magnifique sapin de Céphalonique, âgé de 90 ans et mesurant 2,70 $\mathrm{m}$ de tour.

Dans les plantations, il faut bien se garder de mélanger pied par pied des essences trop diverses, n'ayant ni les mêmes exigences, ni le unême comportement, mais tout en conservant le souci de maintenir avant tout la continuité de l'état boisé et de faire en conséquence des plantations bien appropriées, on peut utilement, dans des massifs boisés de promenade, tenter de ci, de là l'introduction, ne serait-ce qu'en faible quantité, à titre d'essai, de jeunes plants d'espèces très diverses. Une promenade riche en exotiques ne peut que gagner en charme, en pittoresque et aussi en intérêt scientifique.

Dans ses opérations, le reboiseur d'une promenade se doit de chercher toujours à la rendre plus belle, plus agréable, sans jamais se permettre de l'enlaidir, surtout par de fortes trouées intempestives, ne serait-ce que pour quelques années. Qu'il n'oublie jamais que sa plantation va faire partie d'un décor, et que le peuplement qu'il crée sera tout un paysage. Ce ne sera donc pas seulement le souci de créer un peuplement bien adapté au sol et au climat qui doit dicter son choix parmi les essences et dans le groupement, mais aussi de faire quelque chose qui plaise par l'harmonie et le beau.

La nature, en France, bien souvent, nous en donne l'exemple. Pourvu qu'il ait un. peu couru bois et campagnes, le reboiseur se rappellera l'impression ressentie en admirant au printemps le contraste bouleau et résineux. Il ne sera pas resté, insensible à la tâche brillante et orangée qu'apportent, de ci, de là, dans l'uniformité de la verdure, les baies du sorbier des oiseleurs. Il suffit de quelques merisiers, acacias ou cornouillers en fleur pour égayer tout un coin de bois, et sur les sols les plus ingrats, le cytise aux grappes jaunes, associé au pin noir, formera à la fin du printemps un merveilleux ensenble, riant, éclatant de couleur, etc... De même, il ne doit pas craindre d'utiliser à titre secondaire, ne serait-ce que pour 
quelques années, des essences décoratives par leurs fleurs, leur feuillage ou la couleur de leur fût, mélangées par endroits au peuplement principal qu'il crée pour une longue durée.

A Paris, ce souci de faire quelque chose de beau, d'harmonieux sans sortir de la mesure, d'introduire artificiellement la nature en ville sans que la nature y paraisse dépaysée, a certainement guidé autrefois les maitres de l'art du jardinage, qui, il y a cent années, ont créé les promenades de notre capitale.

Voilà un exemple à suivre par tous ceux qui, de nos jours, convaincus de l'utilité de l'arbre, ont le juste désir de lui maintenir ou de lui donner la place et l'importance qu'il mérite dans les espaces verts des agglomérations urbaines.

" La promenade est faite pour le promeneur ». Gardons-nous bien de l'oublier.

Un espace vert doit avant tout plaire à celui qui aura à l'utiliser. Pour atteindre ce but, on peut compter sur l'arbre.

Bien choisi, judicieusement employé, l'arbre y pourvoira.

Eugène Toussaint. 